\title{
Elaboração de um manual ilustrado de exercícios domiciliares para pacientes com hemiparesia secundária ao acidente vascular encefálico (AVE)
}

\author{
Elaboration of an illustrated home exercise manual for stroke patients with hemiparesis
}

Adriana Maria Barsante Santos', Tatiana de Paula Oliveira" ${ }^{2,3,4}$, Maria Elisa Pimentel Piemonte ${ }^{2,5,6}$

\begin{abstract}
RESUMOI A realização de exercícios físicos para pacientes com sequelas motoras pós-acidente vascular encefálico (AVE) é essencial para a recuperação funcional. Programas educacionais podem facilitar a repetição dos exercícios em casa e contribuir para o tratamento. Este trabalho teve como objetivo elaborar e aplicar um manual de exercícios domiciliares para pacientes com AVE. O estudo consistiu em duas etapas: na primeira foi elaborado um manual de exercícios domiciliares (piloto) com fotos e textos simples para facilitar a compreensão. Foi realizada uma avaliação funcional de cada paciente e selecionados os exercícios mais adequados, que deveriam ser feitos em casa. Esse manual foi aplicado a 17 pacientes, 70\% crônicos e 30\% agudos. Após 15 dias, o paciente retornava e era solicitado que reproduzisse os exercícios e informasse o nível de compreensão das fotos e do texto do manual e se sentia dor ao realizá-los. As fotos e os exercícios referidos como difíceis foram revistos, e criada a versão teste que foi aplicada em outros 23 pacientes, dando origem à versão final do manual. A avaliação do manual piloto foi insatisfatória, sendo que apenas $56 \%$ referiram aprovação das fotos e $87 \%$ do texto. Após a reformulação, a compreensão das fotos e do texto alcançou valores acima de $98 \%$. Foi possível obter um manual ilustrado de exercícios domiciliares, de fácil aplicação e compreensão, específico e individualizado, para pacientes com AVE e adaptável aos diferentes quadros motores.
\end{abstract}

Descritores I acidente vascular encefálico; exercício; reabilitação; educação em saúde.

\begin{abstract}
I The practice of exercises for patients with motor sequels post-stroke is essential for the functional recovery. Educational programs can facilitate the repetition of the exercises at home and contribute to the treatment. The objective of this study was to elaborate a home exercise manual for stroke patients. The study consisted of two stages: in the first one, a home exercise manual was made (pilot) with pictures and simple text to facilitate understanding. We made functional assessment of each patient and select the most appropriate exercises that everyone should do at home. This manual has been applied to 17 patients, $70 \%$ chronic and acute 30\%. After 15 days the patient returned and was asked to reproduce the exercises and report the level of understanding of pictures and text of the manual and if felt pain in perform them. Photos and exercises reported such as difficult were reviewed and created a test version that was applied in another 23 patients resulting in the final version of the manual. The evaluation of the manual pilot was unsatisfactory, with only $56 \%$ reported approval of photos and $87 \%$ approval of text. After reformulation, the comprehension of the photos and text reached values above $98 \%$. It was possible to obtain an illustrated manual of home exercises, easy understanding and application specific and personal for stroke patients and adaptable for different motor conditions.
\end{abstract}

Keywords I stroke; exercise; rehabilitation; health education.

Estudo desenvolvido no Departamento de Fisioterapia da Universidade de São Paulo (USP) - São Paulo (SP), Brasil.

'Mestre em Ciências da Reabilitação pela Faculdade de Medicina da USP - São Paulo (SP), Brasil.

${ }^{2}$ Mestre em Neurociências e Comportamento pela USP - São Paulo (SP), Brasil.

3Supervisora de Estágio do Curso de Fisioterapia da USP - São Paulo (SP), Brasil.

${ }^{4}$ Professora do Curso de Fisioterapia da Pontifícia Universidade Católica (PUC) - São Paulo (SP), Brasil.

${ }^{5}$ Doutora em Neurociências e Comportamento da USP - São Paulo (SP), Brasil.

${ }^{6}$ Professora do Curso de Fisioterapia da USP - São Paulo (SP), Brasil. 


\section{INTRODUÇÃO}

O acidente vascular encefálico (AVE) é uma doença encefalovascular causada por interrupção do suprimento sanguíneo no cérebro ${ }^{1}$, responsável por $10 \%$ dos óbitos em todo o mundo ${ }^{2}$. O AVE pode causar sequelas motoras e sensoriais e prejudicar a independência funcional, trazendo impacto negativo na qualidade de $v^{\text {vida }}{ }^{3,4}$. A atuação da fisioterapia é um fator importante para a melhora da qualidade de vida desses pacientes $^{5,6}$. Programas de fortalecimento e condicionamento físico têm demonstrado resultados positivos no que diz respeito aos ganhos de força ${ }^{7,8}$, marcha ${ }^{9}$, mobilidade ${ }^{10}$ e função ${ }^{11} \mathrm{em}$ pacientes pós AVE. A American Heart Association recomenda que esses pacientes realizem atividades físicas regularmente, alegando melhora cardiorrespiratória, motora e funcional ${ }^{12}$.

$\mathrm{O}$ aumento da frequência dos exercícios contribui para a aprendizagem motora, além de otimizar o ganho de força e condicionamento físico com consequente melhora funcional ${ }^{13,14}$. No entanto, o custo financeiro da reabilitação é alto para os pacientes e órgãos de saúde ${ }^{15,16}$. Assim, programas educacionais que ensinem os pacientes a continuar com os exercícios em casa, independentemente, são viáveis e apresentam bom custo-benefício, visto que o número de visitas dos pacientes aos centros de saúde seria reduzido ${ }^{17,18}$.

Porém, na prática clínica observa-se a dificuldade que os pacientes apresentam para se lembrar e executar os exercícios orientados corretamente. Isso ocorre, muitas vezes, devido à natureza da lesão, que proporciona afasias e alterações de memória ${ }^{1}$, o que pode se somar ao baixo nível de escolaridade, considerando a realidade brasileira ${ }^{19}$.

Este trabalho teve como objetivo elaborar e aplicar um manual de exercícios domiciliares para pacientes com AVE.

\section{CASUÍSTICA E MÉTODO}

Participaram do estudo 40 pacientes $(23$ mulheres e 17 homens) com diagnóstico médico de AVE, sendo $70 \%$ com quadro crônico e $30 \%$ agudo.

O critério de inclusão foi apresentar sequelas motoras, sem comprometimento cognitivo severo; os de exclusão foram sequelas exclusivamente sensoriais e ou cerebelares. Os dados demográficos e as características da lesão estão especificados na Tabela 1 . O presente estudo foi aprovado pelo Comitê de Ética do Hospital das Clínicas da Faculdade de Medicina da Universidade de São Paulo e todos os participantes assinaram o Termo de Consentimento Livre e Esclarecido.

$\mathrm{Na} 1^{\text {a }}$ etapa do estudo, um manual piloto foi elaborado, contendo 26 blocos de exercícios baseados nas mais frequentes alterações motoras decorrentes do AVE. Para cada bloco, foram elaboradas três progressões do mesmo movimento: execução ativo-assistida, ativo-livre e ativo-resistida. Nessa etapa, 17 pacientes foram avaliados e, conforme suas sequelas motoras, foram selecionados até cinco exercícios para cada caso, formando, portanto, um conjunto individual de exercícios para cada paciente.

Tabela 1. Dados demográficos e características da lesão nos grupos da 1a e $2^{\mathrm{a}}$ etapas

\begin{tabular}{|c|c|c|c|c|}
\hline \multirow[b]{2}{*}{ Variável } & \multicolumn{2}{|c|}{$1^{\mathrm{a}}$ etapa } & \multicolumn{2}{|c|}{$2^{\mathrm{a}}$ etapa } \\
\hline & Agudos $(n=5)$ & Crônicos (n=12) & Agudos $(n=7)$ & Crônicos ( $n=16)$ \\
\hline \multicolumn{5}{|l|}{ Gênero* } \\
\hline Masculino & $3(60 \%)$ & $1(8 \%)$ & $5(71 \%)$ & $8(50 \%)$ \\
\hline Feminino & $2(40 \%)$ & 11 (92\%) & $2(29 \%)$ & $8(50 \%)$ \\
\hline Idade (anos)** & $57,6(17,2)$ & $59,5(12,2)$ & $42,3(12,6)$ & $63,8(11,6)$ \\
\hline Escolaridade (anos)** & $7(0)$ & $7,1(5,6)$ & $9,9(3,2)$ & $6,1(3,1)$ \\
\hline \multicolumn{5}{|l|}{ Lado da lesão* } \\
\hline Direito & $4(80 \%)$ & $8(67 \%)$ & $3(43 \%)$ & $9(5,6 \%)$ \\
\hline Esquerdo & $1(20 \%)$ & $4(33 \%)$ & $4(57 \%)$ & $7(44 \%)$ \\
\hline \multicolumn{5}{|l|}{ Tipo da lesão* } \\
\hline Isquêmico & $5(100 \%)$ & $10(83 \%)$ & $6(86 \%)$ & 13(81\%) \\
\hline Hemorrágico & $\mathrm{O}(\mathrm{O} \%)$ & $2(17 \%)$ & $1(14 \%)$ & $3(19 \%)$ \\
\hline \multicolumn{5}{|l|}{ Afasia* } \\
\hline Sim & $1(20 \%)$ & $1(8 \%)$ & $3(43 \%)$ & $6(38 \%)$ \\
\hline Não & $4(80 \%)$ & $11(92 \%)$ & $4(57 \%)$ & $10(62 \%)$ \\
\hline
\end{tabular}

Agudos: até e inclusive seis meses de lesão; Crônicos: acima de sete meses de lesão

*dados apresentados em forma de n (frequência)

**dados apresentados em forma de média (desvio-padrão) 
O fisioterapeuta orientava a realização diária de cada exercício e definia o número de repetições.

Após 15 dias, os exercícios eram retomados, corrigidos e progredidos, se fosse o caso. Nesse retorno, era aplicado um questionário em que o paciente avaliava a visualização e a compreensão das fotos, do texto, a presença de dor durante ou após o exercício e a frequência de realização de cada exercício orientado. Conforme a avaliação dos exercícios, aqueles que apresentaram avaliação insatisfatória foram modificados e novos exercícios inseridos, constituindo o manual-teste.

A $2^{a}$ etapa constituiu na aplicação do manual teste em 23 pacientes e repetiu os mesmos procedimentos da $1^{\text {a }}$ etapa (Figura 1 ).

Os resultados obtidos da avaliação dos exercícios nas duas etapas foram analisados e comparados.

\section{Análise estatística}

Foi realizada análise descritiva das variáveis: idade, anos de estudo (média e desvio-padrão), gênero, lado da lesão e presença de afasia.

Os resultados da avaliação dos exercícios obtidos, conforme a aplicação do questionário, foram descritos em termos de distribuição de frequências, e para a avaliação do índice de aprovação dos exercícios do manual piloto e do manual teste, foi utilizado o teste $\chi^{2}$, que permitiu comparar a frequência de aprovação observada com a esperada. Foi considerada como esperada a aprovação de $100 \%$ nos itens de avaliação da foto, do texto e da ausência de dor. Para comparar o índice de aprovação do manual piloto com o do manual teste, foi utilizado o teste de Wilcoxon. O nível de significância foi estabelecido como $\alpha=0,05$.

\section{RESULTADOS}

A Tabela 1 mostra os dados demográficos e as características da lesão nos grupos da $1^{\mathrm{a}}$ e $2^{\mathrm{a}}$ etapas.

\section{Avaliação do manual}

Os resultados da $1^{\mathrm{a}}$ etapa, referentes à aplicação e avaliação do manual piloto, estão especificados na Tabela 2. Observa-se que a avaliação das fotos e do texto foi inferior aos valores esperados. O número de repetições foi fácil identificar, atingindo o esperado (100\%). A presença de dor foi observada em 9\%, durante a realização
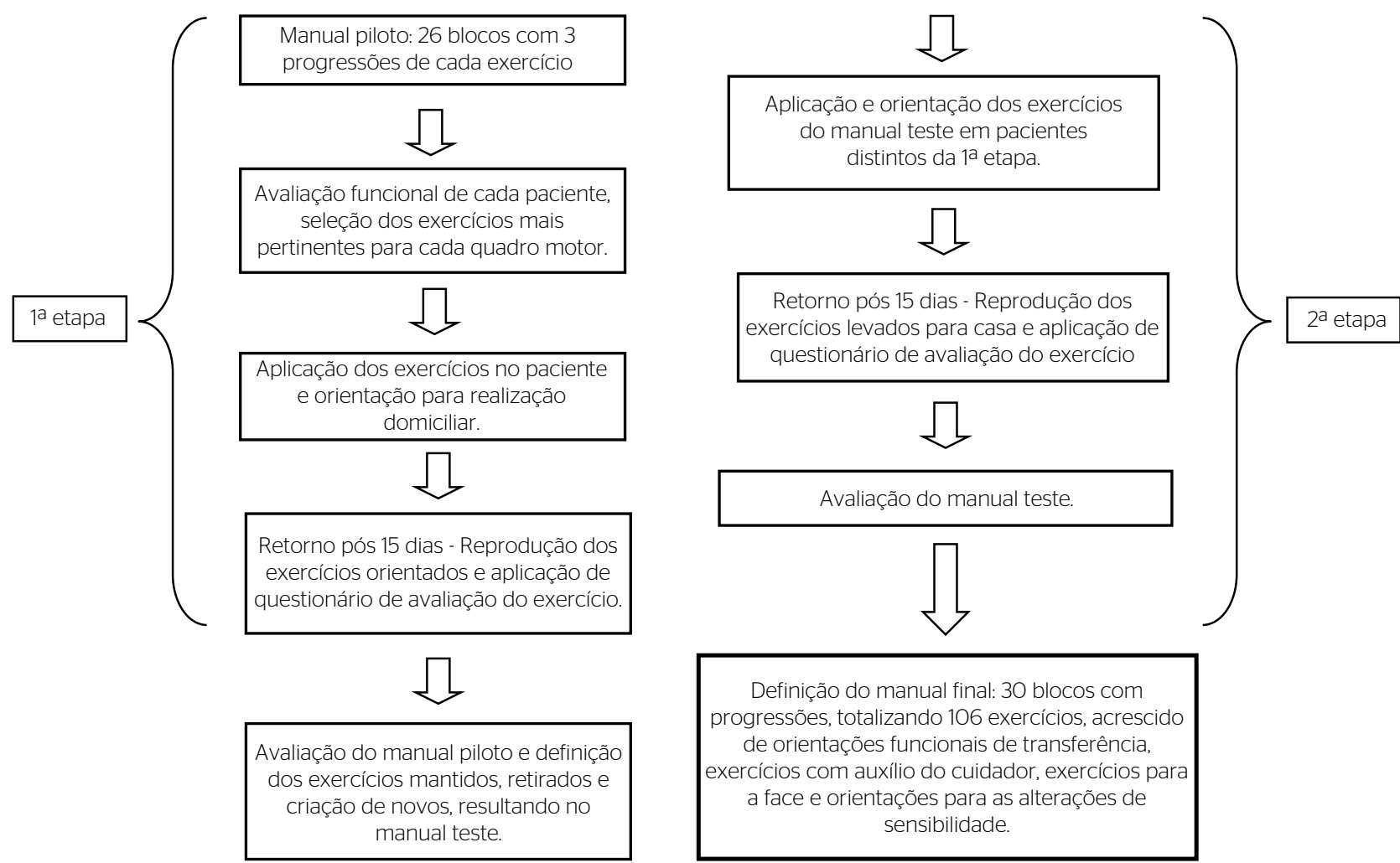


\begin{tabular}{|c|c|c|c|c|}
\hline & Aprovação observada & Aprovação esperada & $\chi^{2}$ & Valor $\mathrm{p}$ \\
\hline \multicolumn{5}{|l|}{ Manual piloto $(\mathrm{n}=17)(\%)$} \\
\hline \multicolumn{5}{|l|}{ Foto } \\
\hline Visualização & 56 & 100 & 48,42 & $<0,0001^{\star}$ \\
\hline Compreensão do posicionamento & 59 & & & \\
\hline Compreensão do movimento & 65 & & & \\
\hline \multicolumn{5}{|l|}{ Texto } \\
\hline Compreensão do texto & 87 & 100 & 12,35 & $<0,01^{*}$ \\
\hline Compreensão do posicionamento & 79 & & & \\
\hline Compreensão do movimento & 75 & & & \\
\hline \multicolumn{5}{|l|}{ Manual teste $(n=23)(\%)$} \\
\hline \multicolumn{5}{|l|}{ Fotos } \\
\hline Visualização & 100 & 100 & 0,045 & 0,97 \\
\hline Compreensão do posicionamento & 98.5 & & & \\
\hline Compreensão do movimento & 98.5 & & & \\
\hline \multicolumn{5}{|l|}{ Texto } \\
\hline Compreensão do texto & 100 & 100 & 0,050 & 0,99 \\
\hline Compreensão do posicionamento & 99 & & & \\
\hline Compreensão do movimento & 98 & & & \\
\hline
\end{tabular}

$\chi^{2}$ : teste Qui-quadrado; "estatisticamente significante; $p<0,05$

dos exercícios do manual, e 5\% após, valores não significativos $\left(\chi^{2}=1,060 ; p=0,30\right)$. Quanto à frequência de realização do exercício em casa, $80 \%$ dos pacientes realizaram os exercícios propostos três ou mais vezes (média de 3,75$)$ na última semana.

Diante desses resultados da aplicação do manual piloto, os exercícios que apresentaram avaliação negativa foram retirados ou modificados e novos exercícios foram propostos, constituindo no manual teste.

Os resultados da $2^{a}$ etapa, referentes à aplicação e àvaliação do manual piloto, são apresentados na Tabela 2. Observa-se que a avaliação das fotos e do texto atingiu os valores esperados. A presença de dor foi observada apenas durante a realização de 3\% dos exercícios do manual piloto, com valores não significativos $\left(\chi^{2}=0,900 ; p=0,34\right)$. Quanto à frequência de realização do exercício em casa, 91\% dos pacientes realizaram os exercícios propostos três ou mais vezes (média de 4,01) na última semana.

Todos os blocos de exercícios tiveram pelo menos dois exercícios (85\%) avaliados por pacientes afásicos ou com grau de escolaridade inferior a cinco anos. Esses exercícios avaliados receberam 100\% de aprovação desses pacientes.

Ao final da aplicação do manual teste, os resultados de sua avaliação foram comparados aos resultados da avaliação do manual piloto. Houve aumento significativo na aprovação de todos os quesitos avaliados nas fotos $(\mathrm{p}<0,001)$ e no texto $(\mathrm{p}<0,001)$ do manual teste.

Diante da avaliação positiva do manual teste, esse foi considerado o manual final que resultou em 106 exercícios, com progressões de dificuldade, tendo execução ativo-assistida, ativa e ativo-resistida, podendo, ainda, variar nas diferentes posturas, como: deitado, sentado e em pé.

Os exercícios do manual final foram divididos em 30 blocos, contemplando os seguintes movimentos: extensão, adução e abdução de quadril, tríplice flexão, fortalecimento de abdominais, mobilização de tronco, extensão, flexão e controle de joelho, alongamento de tríceps sural, dorsiflexão, plantiflexão e eversão de tornozelo, mobilização de cabeça, mobilização/alongamento de membro superior, abdução e extensão de ombro, extensão e flexão de cotovelo, pronação e supinação de antebraço, alongamento/mobilização e extensão de punho, extensão, flexão e abdução dos dedos da mão, intrínsecos dos dedos da mão, coordenação de movimentos da mão, descarga de peso, apoio e oscilação na marcha. O Quadro 1 ilustra um dos blocos de exercícios do manual final.

\section{DISCUSSÃO}

Semelhante à ideia deste estudo, pesquisadores alemães e holandeses desenvolveram um sistema de computador simples e barato para ser utilizado em casa, que direciona a execução do exercício, com intuito de aumentar sua frequência, otimizando a reabilitação de membros superiores após sequela de $\mathrm{AVE}^{18}$. 
Quadro 1. Exemplo de um bloco de exercícios (retiradas as fotos). Cada exercício indica o posicionamento inicial e durante a realização

\section{EXEMPLO: EXERCICÍOS PARA A EXTENSÃO DE COTOVELO \\ EXERCICIO A \\ Posicionamento Inicial: Deitado de barriga para cima sobre uma superfície não muito mole. Pernas dobradas. Costas apoiadas desde o pescoço até o bumbum. Braços ao longo do corpo e cotovelo dobrado. \\ a) estique o cotovelo fraco \\ b) volte à posição inicial; \\ c) realize o movimento __ vezes. \\ Descanse. Repita o movimento __ vezes. \\ EXERCICIO B \\ Posicionamento Inicial: Deitado de barriga para cima sobre uma superfície não muito mole. Pernas esticadas. Costas apoiadas desde o pescoço até o bumbum. Braços junto ao corpo. \\ a) coloque a mão fraca na orelha do lado bom, apoiando o braço fraco com a mão boa; \\ b) estique o cotovelo fraco o máximo que puder, levando a mão para cima; \\ c) volte a mão fraca na orelha; \\ d) realize o movimento __ vezes. Descanse. Repita o exercício _ vezes.}

\section{EXERCICIO C:}

Posicionamento Inicial: Deitado de barriga para cima sobre uma superfície não muito mole. Pernas dobradas. Costas apoiadas desde o pescoço até o bumbum. Braços junto ao corpo.

a) pegue um elástico forte, dê um nó nas pontas formando um círculo;

b) coloque o elástico preso ao ombro bom;

c) leve a mão fraca no ombro do lado bom, passe a mão fraca dentro do elástico e apoie o braço fraco com a mão boa;

d) fixe o elástico no punho;

e) enrole um pedaço de pano no punho para não machucar a pele;

f) estique o cotovelo fraco o máximo que puder, esticando o elástico; g) volte a mão fraca no ombro. Descanse. Repita o exercício _ _ vezes

Há outros trabalhos na literatura que propõem programas de exercícios domiciliares, porém com diferentes intervenções e propósitos. Duncan et al. ${ }^{20}$ implantaram um programa de exercícios domiciliares três vezes por semana, durante oito semanas, em pacientes de AVE leve e moderado, observando melhora funcional de membros inferiores. Já Olney et al. ${ }^{21}$ compararam um programa de exercícios supervisionados com não supervisionados (apenas com uma instrução prévia) e observaram benefícios físicos imediatos e após um ano em ambos os programas. Outro estudo, em 2009, observou se um programa de exercícios domiciliares melhora a força, o desempenho físico e a capacidade funcional de pacientes com AVE crônico ${ }^{22}$. Nesses estudos, a preocupação maior foi avaliar a melhora funcional e pouco é detalhado sobre a escolha e a orientação dos exercícios que deviam ser realizados em casa.
Desse modo, não há, na literatura, guias que proponham a elaboração de um manual para pacientes com AVE nos moldes deste estudo, sendo, o procedimento elaborado segundo a experiência clínica dos pesquisadores, e baseados nas alterações motoras presentes na hemiparesia ${ }^{23,24}$. Para a detecção dos pontos não aplicáveis, foi elaborado um manual piloto. A três progressões do mesmo movimento, execução ativo-assistida, ativa e ativo-resistida, tiveram como objetivo definir o nível de dificuldade de cada exercício proposto, englobando, assim, pacientes com diferentes graus de necessidade e podendo progredir o nível de acordo com a evolução do paciente.

Para isso, a avaliação funcional de todos os pacientes foi primordial, pois pôde-se entender as principais deficiências e atuar pontualmente com os exercícios, tendo em vista que quanto mais específico é o tratamento, maior será o aprendizado motor, e, consequentemente, maior será a recuperação do paciente ${ }^{13}$. Essa avaliação funcional identificou os principais acometimentos ${ }^{23}$,e o formato do manual proposto permitiu atender exclusivamente as demandas de cada paciente, uma vez que era possível selecionar um exercício de execução ativo-assistida para um grupo muscular de maior limitação e um exercício de execução ativo-resistida para outro grupo, caso já apresentasse melhor desempenho. Além disso, esse formato viabilizou que o paciente levasse a folha do exercício selecionado para casa, o que visou maior adesão ao tratamento ${ }^{25}$.

$\mathrm{Na}$ confecção do manual, a avaliação feita pelos questionários e o perfil da amostra, englobando pacientes afásicos e com baixo nível de escolaridade, direcionaram a elaboração dos exercícios às necessidades dos pacientes, resultando em exercícios simples, de fácil posicionamento e compreensão, com textos e fotos claras e objetivas. Em cada exercício foi indicado como realizar o movimento adequadamente, o que deveria sentir durante sua execução, o que não deveria ser feito e o número de repetições. Dessa forma, foi possível obter um manual aplicável e de fácil reprodução, englobando exercícios de alongamento, fortalecimento e treinamentos funcionais, essenciais para a recuperação do paciente com $\mathrm{AVE}^{10}$.

Foi orientada a realização diária domiciliar, tendo em vista que a repetição influencia no aprendizado motor ${ }^{13}$. Foi observado que $91 \%$ dos pacientes realizaram os exercícios orientados mais de três vezes por semana, mantendo, portanto, alta frequência de treinamento, o que pode resultar em ganhos funcionais e maior qualidade de vida ${ }^{6,14,26}$.

Sobre o tempo de reavaliação para analisar a adesão ao exercício, não há suporte na literatura. Os estudos divergem: Figueredo et al. ${ }^{17}$ optaram por reavaliar a adesão 
aos exercícios propostos a pacientes ambulatoriais submetidos à aplicação de toxina botulínica pós-trinta dias. Já Caglar et al. ${ }^{27}$ reavaliaram pacientes com doença de Parkinson após dois meses da instrução dos exercícios domiciliares. Esse último, no entanto, apresentou intuito diferente deste estudo, pois buscou avaliar a melhora funcional e não a adesão aos exercícios orientados.

\section{CONCLUSÃO}

O processo de elaboração permitiu a confecção de um manual de exercícios domiciliares ilustrado, específico e individualizado para pacientes com sequelas de AVE, com progressões de exercícios que preveem a evolução funcional de pacientes agudos e crônicos. O manual mostrou-se de fácil aplicação e de boa reprodução pelo paciente.

\section{REFERÊNCIAS}

1 Smith WS, Hauser SL, Easton JD. Doenças Cerebrovasculares. In: Braunwald E, Fauci AS, Kasper DL, Hauser SL, Longo DL, Jameson JL. Harrison Medicina Interna. 15a ed. Rio de Janeiro: Mc Graw -Hill; 2002. p. $2517-40$

2 Who.int [Internet]. The Atlas of Heart Disease and stroke [cited 2007 May 25]. Available from: http://www.who.int/cardiovascular_diseases/ en/cvd_atlas_01_types.pdf

3 Kelly-Hayes M, Robertson JT, Broderick JP, Duncan PW, Hershey LA, Roth EJ, et al. The American Heart Association Stroke Outcome Classification. Stroke. 1998;29(6):1274-80.

4 Martins T, Ribeiro JP, Garrett C. Disability and quality of life of stroke survivors: evaluation nine months after discharge. Rev Neurol. 2006:42(11):655-9.

5 Kalra L, The influence of stroke unit rehabilitation on functional recovery from stroke. Stroke. 1994;25(4):821-5.

6 Chen MD, Rimmer JH. Effects of exercise on quality of life in stroke survivors: a meta-analysis. Stroke. 2011;42(3):832-7.

7 Morris SL, Dodd KJ, Morris ME. Outcomes of progressive resistance strength training following stroke: a systematic review. Clin Rehabil. 2004:18(1):27-39.

8 Mercer VS, Chang SH, Williams CD, Noble K, Vance AW. Effects of an exercise program to increase hip abductor muscle strength and improve lateral stability following stroke: a single subject design. J Geriatr Phys Ther. 2009;32(2):50-9.

9 Saunders DH, Greig CA, Young A, Mead GE. Physical fitness training for stroke patients. Stroke. 2004;35(9):2235.

10 Marigold DS, Eng JJ, Dawson AS, Inglis JT, Harris JE, Gylfadottir S. Exercise leads to faster postural reflexes, improved balance and mobility, and fewer falls in older persons with chronic stroke. J Am Geriatr Soc. 2005;53(3):416-23.

11 Van Peppen RP, Kwakkel G, Wood-Dauphinee S, Hendriks HJ, Van Der Wees PJ, Dekker J. The impact of physical therapy on functional outcomes after stroke: what's the evidence? Clin Rehabil. 2004:18(8):833-62.

12 Gordon NF, Gulanick M, Costa F, Fletcher G, Franklin BA, Roth EJ, et al. American Heart Association Council on Clinical Cardiology, Subcommittee on Exercise, Cardiac Rehabilitation, and Prevention; the Council on Cardiovascular Nursing; the Council on Nutrition, Physical Activity, and Metabolism; and the Stroke Council. Physical activity and exercise recommendations for stroke survivors: an American Heart Association scientific statement from the Council on Clinical Cardiology, Subcommittee on Exercise, Cardiac Rehabilitation, and Prevention; the Council on Cardiovascular Nursing; the Council on Nutrition, Physical Activity, and Metabolism; and the Stroke Council. Circulation. 2004:109(16):2031-41.

13 Woollacott MH, Cook AS. Controle motor: teoria e aplicações. 2a ed. Barueri: Manole; 2003.

14 Cooke1 EV, Mares K, Clark A, Tallis RC, Pomeroy VM. The effects of increased dose of exercise-based therapies to enhance motor recovery after stroke: a systematic review and meta-analysis. BMC medicine. 2010;13:8-60.

15 Porsdal V. Boysen G. Costs of health care and social services during the first year after ischemic stroke. Int J Technol Assess Health Care. 1999;15(3):573-84.

16 Saka O, McGuire A. Wolfe C. Cost of stroke in the United Kingdom. Age Ageing. 2009;38(1):27-32.

17 Figueiredo MMP, Barbosa MCC, Moreira MCS. Avaliação de um manual de exercícios domiciliares para pacientes externos de um ambulatório de bloqueio neuromuscular. Acta Fisiátrica. 2005:12(1):7-10.

18 Willmann RD, Lanfermann G, Saini P, Timmermans A, Vrugt J, Winter S. Home stroke rehabilitation for the upper limbs. Conf Proc IEEE Eng Med Biol Soc. 2007:4015-8.

19 Instituto Brasileiro de Geografia e Estatística (IBGE) - Censo 2000 [Internet] Brasília. Ministério do Planejamento, Orçamento e Gestão. [cited 2008 Jan 18]. Available from: http://www.ibge.gov.br/home/ mapa_site/mapa_site.php\#download

20 Duncan P, Richards L, Wallace D, Stoker-Yates J, Pohl P, Luchies C, et al. A randomized, controlled pilot study of a home-based exercise program for individuals with mild and moderate stroke. Stroke. 1998;29(10):2055-60.

21 Olney SJ, Nymark J, Brouwer B, Culham E, Day A, Heard J, et al. A randomized controlled trial of supervised versus unsupervised exercise programs for ambulatory stroke survivors. Stroke. 2006:37(2):476-81.

22 Mercer VS, Chang SH, Williams CD, Noble K, Vance AW. Effects of an exercise program to increase hip abductor muscle strength and improve lateral stability following stroke: a single subject design. J Geriatr Phys Ther. 2009;32(2):50-9.

23 Ryerson SD. Hemiplegia. In: Umphred DA. Reabilitação neurológica. 4a ed. Barueri: Manole; 2004. p. 782-830.

24 Davies PM. Passos a seguir. Um manual para o tratamento da hemiplegia no adulto. São Paulo: Manole; 1996.

25 Valim V. Benefícios dos exercícios físicos na fibromialgia. Rev Bras Reumatol. 2006;46(1):49-55.

26 Lustyk MK, Widman L, Paschane AA, Olson KC. Physical activity and quality of life: assessing the influence of activity frequency, intensity, volume, and motives. Behav Med. 2004;30(3):124-31.

27 Caglar AT, Gurses HN, Mutluay FK, Kiziltan G. Effects of home exercises on motor performance in patients with Parkinson's disease. Clin Rehabil. 2005 19(8):870-7. 